LEITE, G.L.D.; SANTOS, M.C.; ROCHA, S.L.; COSTA, C.A.; ALMEIDA, C.I.M. Intensidade de ataque de tripes, de alternaria e da queima-das-pontas em cultivares de cebola. Horticultura Brasileira, Brasília, v.22, n.1, p. 151-153, jan-mar 2004.

\title{
Intensidade de ataque de tripes, de alternaria e da queima-das-pontas em cultivares de cebola
}

\author{
Germano L.D. Leite*; Marília Cristina dos Santos; Silma L. Rocha; Cândido A. da Costa; Chrystian I. \\ Maia e Almeida \\ Universidade Federal de Minas Gerais, NCA, Fitotecnia, C. Postal 135, 39404-006 Montes Claros-MG; E-mail: gldleite@nca.ufmg.br; \\ *Autor Correspondente
}

\section{RESUMO}

Avaliou-se a intensidade de ataque de Thrips tabaci Lind., a incidência de Alternaria porri (Ellis) e Botrytis squamosa J.C. Walker em nove cultivares de cebola [Aurora, Primavera, CNPH 6400, Crioula Alto-Vale, Vale-Ouro (IPA 11), Franciscana (IPA-16), Piraouro, Conquista e Serrana], num experimento no delineamento em blocos casualizados com cinco repetições. Foram feitas avaliações semanais, do transplantio até a colheita, em dez plantas/parcela do número de $T$. tabaci presente na bainha das folhas bem como da percentagem de área foliar danificada pelo mesmo a partir da primeira folha expandida e também da presença ou da ausência de lesões causadas por $A$. porri e $B$. squamosa. A cv. Franciscana mostrou-se mais resistente em relação às demais ao ataque de T. tabaci. Não houve diferença significativa de plantas afetadas por A. porri e B. squamosa. T. tabaci e A. porri foram ambos afetados positiva e negativamente pela temperatura e pela umidade relativa do ambiente, respectivamente.

Palavras-chave: Thrips tabaci, Alternaria porri, Botrytis squamosa, Allium cepa.

\begin{abstract}
Intensity of attacks of thrips, purple blotch and gray mold on onion cultivars

The intensity of attack of T. tabaci and incidence of Alternaria porri (Ellis) Cif and Botrytis squamosa J.C. Walker in nine onion cultivars [Aurora, Primavera, CNPH 6400, Crioula Alto-Vale, ValeOuro (IPA 11), Franciscana (IPA-16), Piraouro, Conquista and Serrana] was evaluated, in an experiment in randomized blocks design, with five replications. Weekly evaluations were done from the transplanting until harvesting date, in ten plants/parcel of the number of $T$. tabaci on the leaves, the percentage of damaged foliar area from the first expanded leaf up and the presence or absence of injuries caused by $A$. porri and B. squamosa. The cv. Franciscana was more resistant to the attack of T. tabaci in comparison to the other onion cultivars. There was no significant difference between cvs for A. porri and Botrytis squamosa resistance. T. tabaci and A. porri were influenced positively and negatively by temperature and relative humidity, respectively.
\end{abstract}

Keywords: Thrips tabaci, Alternaria porri, Botrytis squamosa, Allium cepa.

(Recebido para publicação em 17 de fevereiro de 2003 e aceito em 28 de novembro de 2003)

\begin{abstract}
$\mathrm{A}$ cebola (Allium cepa) L., de origem asiática, foi trazida ao Brasil pelos portugueses, sendo cultivada em quase todo o país, principalmente nas regiões Sul e Sudeste (Filgueira, 2000). O Norte de Minas Gerais, mais precisamente, o vale do São Francisco, é importante produtor dessa espécie. Esta região, de transição para o clima semiárido (quente e seco), que favorece a maturação do bulbo (Filgueira, 2000). A cultura é afetada por pragas como tripes [Thrips tabaci Lind (Thysanoptera: Thripidae)] (Gallo et al., 2002) e doenças, destacando-se alternaria [Alternaria porri (Ellis)] (Zambolim et al., 2000) e queima-das-pontas (Botrytis squamosa J.C. Walker) (Agrios, 1988; Kimati et al., 1997). O ataque dessa praga e doenças causam grandes perdas na produção e podem inviabilizar a cebolicultura, sendo importante identificar cultivares mais resistente.
\end{abstract}

Este trabalho teve como objetivo avaliar a resistência de nove cultivares de cebola a tripes, alternaria e queimadas-pontas, sob sistema convencional de cultivo no Norte de Minas Gerais.

\section{MATERIAL E MÉTODOS}

O experimento foi desenvolvido de 04/06 a 30/08/02 na UFMG, em Montes Claros, longitude de $43^{\circ} 53^{\prime} \mathrm{W}$, latitude de $16^{\circ} 43^{\prime} \mathrm{S}$ e altitude: $650 \mathrm{~m}$. Segundo a classificação de Köppen, o clima é o Aw: tropical de savana, inverno seco e verão chuvoso.

$\mathrm{O}$ delineamento foi em blocos casualizados com cinco repetições, onde cada parcela consistiu de cinco fileiras de plantas com $3 \mathrm{~m}$ de comprimento e 1 $\mathrm{m}$ de largura (unidade experimental de $3 \mathrm{~m}^{2}$ ), totalizando $135 \mathrm{~m}^{2}$ de área total. A área útil do experimento foi de 0,60 $\mathrm{m}^{2}$ por parcela, constituída pela fileira central. Os tratamentos constituíram-se de nove cultivares de cebola [Aurora, Primavera, CNPH 6400, Crioula AltoVale, Vale-Ouro (IPA 11), Franciscana (IPA-16), Piraouro, Conquista e Serrana]. O transplantio das mudas foi realizado 45 dias após a semeadura em espaçamento de $20 \times 7 \mathrm{~cm}$, irrigadas por micro-aspersão e, sendo a partir desse período iniciadas as avaliações.

Avaliou-se, semanalmente, do transplantio até a colheita, por meio de contagem direta, o número de tripes presentes na bainha das folhas bem como a percentagem de área foliar danificada pelo mesmo a partir da primeira folha expandida em dez plantas/parcela, adaptando as metodologias de Gonçalves (1997), Edelson et al. (1986), Shelton et al. (1987) e de Domiciano et al. (1993). Nessas mesmas amostras foram também avaliada a presença ou ausência de lesões causadas por alternaria e por queima das pontas. Os dados foram 
G. L. D. Leite et al.

Tabela 1. Número e percentagem de área foliar de nove cultivares de cebola afetadas por Thrips tabaci. Montes Claros, UFMG, 2002.

\begin{tabular}{lcc}
\hline \multicolumn{1}{c}{ Cultivares } & Número de tripes/planta & Área foliar danificada (\%) \\
\hline Aurora & $3,83 \mathrm{~b}$ & $12,11 \mathrm{~b}$ \\
Primavera & $3,51 \mathrm{c}$ & $12,21 \mathrm{~b}$ \\
CNPH-6400 & $3,87 \mathrm{~b}$ & $15,62 \mathrm{a}$ \\
Crioula Alto-Vale & $5,97 \mathrm{a}$ & $11,80 \mathrm{~b}$ \\
Vale-Ouro & $3,10 \mathrm{c}$ & $11,03 \mathrm{~b}$ \\
Franciscana & $3,00 \mathrm{c}$ & $9,35 \mathrm{c}$ \\
Piraouro & $2,82 \mathrm{C}$ & $10,97 \mathrm{~b}$ \\
Conquista & $4,03 \mathrm{~b}$ & $17,68 \mathrm{a}$ \\
Serrana & $3,58 \mathrm{~b}$ & $15,25 \mathrm{a}$ \\
\hline
\end{tabular}

Médias seguidas pela mesma letra nas colunas não diferem, entre si, pelo teste de Scott Knott a 5\% de probabilidade.

Tabela 2. Número médio/planta e de percentagem de área foliar de cebola danificada por Thrips tabaci, percentagem de plantas de cebola danificadas por Alternaria porri e Botrytis squamosa. Montes Claros, UFMG, 2002.

\begin{tabular}{lccrrrr}
\hline \multirow{2}{*}{ Cultivares } & \multicolumn{3}{c}{ No. de T. tabacilplanta } & \multicolumn{2}{c}{ Área foliar (\%) danificada por T. tabaci } \\
\cline { 2 - 7 } & Junho & Julho & \multicolumn{1}{c}{ Agosto } & Junho & Julho & Agosto \\
\hline Aurora & $0,00 \mathrm{~B}$ & $1,37 \mathrm{~B}$ & $6,56 \mathrm{~A}$ & $1,04 \mathrm{~B}$ & $6,07 \mathrm{~B}$ & $17,98 \mathrm{~A}$ \\
Primavera & $0,02 \mathrm{~B}$ & $1,11 \mathrm{~B}$ & $6,13 \mathrm{~A}$ & $7,60 \mathrm{~B}$ & $8,95 \mathrm{~B}$ & $15,74 \mathrm{~A}$ \\
CNPH-6400 & $0,02 \mathrm{~B}$ & $1,74 \mathrm{~B}$ & $6,34 \mathrm{~A}$ & $7,86 \mathrm{~B}$ & $7,90 \mathrm{~B}$ & $22,58 \mathrm{~A}$ \\
Crioula Alto-Vale & $0,00 \mathrm{C}$ & $2,31 \mathrm{~B}$ & $10,07 \mathrm{~A}$ & $5,28 \mathrm{~B}$ & $8,11 \mathrm{~B}$ & $16,05 \mathrm{~A}$ \\
Vale-Ouro & $0,00 \mathrm{~B}$ & $1,49 \mathrm{~B}$ & $5,02 \mathrm{~A}$ & $5,00 \mathrm{~B}$ & $5,15 \mathrm{~B}$ & $16,94 \mathrm{~A}$ \\
Franciscana & $0,00 \mathrm{~B}$ & $1,07 \mathrm{~B}$ & $5,15 \mathrm{~A}$ & $7,44 \mathrm{~B}$ & $5,15 \mathrm{~B}$ & $13,10 \mathrm{~A}$ \\
Piraouro & $0,00 \mathrm{~B}$ & $0,80 \mathrm{~B}$ & $4,99 \mathrm{~A}$ & $6,06 \mathrm{~B}$ & $5,00 \mathrm{~B}$ & $16,73 \mathrm{~A}$ \\
Conquista & $0,00 \mathrm{C}$ & $2,13 \mathrm{~B}$ & $6,35 \mathrm{~A}$ & $7,60 \mathrm{~B}$ & $10,26 \mathrm{~B}$ & $25,64 \mathrm{~A}$ \\
Serrana & $0,00 \mathrm{C}$ & $1,83 \mathrm{~B}$ & $5,71 \mathrm{~A}$ & $7,25 \mathrm{~B}$ & $8,21 \mathrm{~B}$ & $22,40 \mathrm{~A}$ \\
\hline
\end{tabular}

\begin{tabular}{lcccccc}
\hline \multirow{2}{*}{ Cultivares } & \multicolumn{3}{c}{ Plantas danificadas (\%) } & por A. porri & \multicolumn{3}{c}{ Plantas danificadas (\%) por B. squamose } \\
\cline { 2 - 7 } & Junho & Julho & Agosto & Junho & Julho & Agosto \\
\hline Aurora & $0,00 \mathrm{~B}$ & $2,00 \mathrm{~B}$ & $38,00 \mathrm{~A}$ & $0,00 \mathrm{~B}$ & $39,00 \mathrm{~A}$ & $36,00 \mathrm{~A}$ \\
Primavera & $0,00 \mathrm{~B}$ & $0,00 \mathrm{~B}$ & $34,00 \mathrm{~A}$ & $0,00 \mathrm{~B}$ & $40,00 \mathrm{~A}$ & $36,00 \mathrm{~A}$ \\
CNPH-6400 & $0,00 \mathrm{~B}$ & $1,00 \mathrm{~B}$ & $37,00 \mathrm{~A}$ & $0,00 \mathrm{~B}$ & $20,00 \mathrm{~A}$ & $34,00 \mathrm{~A}$ \\
Crioula Alto-Vale & $0,00 \mathrm{~B}$ & $1,00 \mathrm{~B}$ & $33,00 \mathrm{~A}$ & $0,00 \mathrm{~B}$ & $47,00 \mathrm{~A}$ & $31,00 \mathrm{~A}$ \\
Vale-Ouro & $0,00 \mathrm{~B}$ & $0,00 \mathrm{~B}$ & $34,00 \mathrm{~A}$ & $0,00 \mathrm{~B}$ & $34,00 \mathrm{~A}$ & $32,00 \mathrm{~A}$ \\
Franciscana & $0,00 \mathrm{~B}$ & $0,00 \mathrm{~B}$ & $39,00 \mathrm{~A}$ & $0,00 \mathrm{~B}$ & $36,00 \mathrm{~A}$ & $32,00 \mathrm{~A}$ \\
Piraouro & $0,00 \mathrm{~B}$ & $0,00 \mathrm{~B}$ & $57,00 \mathrm{~A}$ & $0,00 \mathrm{~B}$ & $32,00 \mathrm{~A}$ & $32,00 \mathrm{~A}$ \\
Conquista & $0,00 \mathrm{~B}$ & $1,00 \mathrm{~B}$ & $40,00 \mathrm{~A}$ & $0,00 \mathrm{~B}$ & $37,00 \mathrm{~A}$ & $32,00 \mathrm{~A}$ \\
Serrana & $0,00 \mathrm{~B}$ & $1,00 \mathrm{~B}$ & $45,00 \mathrm{~A}$ & $0,00 \mathrm{~B}$ & $42,00 \mathrm{~A}$ & $36,00 \mathrm{~A}$ \\
\hline
\end{tabular}

Médias seguidas pela mesma letra nas linhas não diferem, entre si, pelo teste de Scott Knott a 5\% de probabilidade.

submetidos à análise de variância, teste de média de Scott Knott e à correlação de Pearson a 5\% de significância.

\section{RESULTADOS E DISCUSSÃO}

Observou-se maior número de tripes na cultivar Crioula Alto-Vale em relação às demais (Tabela 1). Contudo, a maior percentagem de área foliar danificada foi detectada nas cultivares Conquista, $\mathrm{CNPH}$ 6400 e Serrana, seguidas por Primavera, Aurora e Crioula Alto-Vale e por ValeOuro (IPA 11), Piraouro e Franciscana (IPA-16) (Tabela 1). Esse fato, possivelmente, se deve à maior suscetibilidade ao ataque de tripes das cultivares $\mathrm{CNPH}$ 6400, Conquista e Serrana em relação às demais, propiciando maior número de lesões nas folhas. Já a cultivar Franciscana (IPA-16) apresentou menor número de tripes/planta e menor percentagem de área foliar danificada, mostrando-se mais resistentes ao ataque desse inseto. 
Observou-se aumento populacional de tripes em todas as cultivares a partir de julho (Tabela 2), possivelmente em função das relações entre umidade relativa (UR) e da temperatura (T) do ar observadas no meses de cultivo [(Junho: $\mathrm{T}=20,73^{\circ} \mathrm{C} ; \mathrm{UR}=58,47 \%$ ), (Julho: $\mathrm{T}=21,38^{\circ} \mathrm{C} ; \mathrm{UR}=56,47 \%$ ), (Agosto: $\left.\left.\mathrm{T}=22,79^{\circ} \mathrm{C} ; \mathrm{UR}=44,88 \%\right)\right]$, já que o aumento da temperatura $(\mathrm{r}=0,99)$ (Lall \& Singh, 1968; Gonçalvez 1997) e a redução da umidade relativa $(\mathrm{r}=-0,99)$ (Lall e Singh, 1968) aceleram o ciclo biológico do $T$. tabaci, favorecendo o seu incremento populacional. O número máximo de tripes/planta foi observado na cv. Crioula Alto-Vale $(10,07 \pm 1,74)$ no mês de agosto, mas ficando abaixo do nível de controle (15 tripes/planta) (Gonçalves, 1997).

As lesões ocasionadas por tripes podem também favorecer a ocorrência de alternaria e da queima-das-pontas (Zambolim et al., 2000). Porém, não se detectou diferença estatística significativa entre as cultivares de cebola quanto a percentagem de plantas afetadas por alternaria $(20,00 \pm 1,00)$ e queima-das-pon$\operatorname{tas}(35,00 \pm 1,00)$. Observou-se maior percentagem de plantas afetadas por alternaria a partir de julho, fato não observado para a queima-das-pontas (Tabela 2), possivelmente devido ao aumento de temperatura e da redução da umidade relativa. A incidência de alternaria é melhor favorecido em altas temperaturas $(\mathrm{r}=0,95)$, porém sob baixa umidade relativa do $\operatorname{ar}(\mathrm{r}=-0,98)$, a doença não se desenvolve podendo surgir manchas brancas estéreis (Zambolim et al., 2000). Temperaturas relativamente baixas e alta umidade relativa são fatores que predispõem a queima-das-pontas (Zambolim et al., 2000). Contudo, neste trabalho, não se detectou correlação significativa desses elementos climáticos sobre queima-das-pontas.

Acv. Franciscana foi a mais resistente ao ataque do tripes, pois apresentou menor número de tripes/planta e menor percentagem de lesões foliares. O Norte de Minas Gerais é um promissor produtor de cebola já que o clima quente e seco não favorece o estabelecimento de alternaria e de queima-das-pontas.

\section{LITERATURA CITADA}

AGRIOS, G.N. Plant Pathology. 3rd ed. San Diego: Academic Press, Inc., 1988. 803 p.

DOMICIANO, N.L.; OTA, A.Y.; TEDARDI, C.R. Momento adequado para controle químico de tripes, Thrips tabaci Lindeman, 1888 em cebola, Allium cepa L. Anais da Sociedade Entomológica do Brasi, Londrina, v. 22, p. 71-76, 1993.
EDELSON, J.V.; CARTWRIGHT, B; ROYER, T.A. Distribution and impact of Thrips tabaci (Thysanoptera: Thripidae) on onion. Journal Economic Entomology, v.79, p.502-505, 1986.

FILGUEIRA, F.A.R. Novo manual de olericultura; Agrotecnologia moderna na produção e comercialização de hortaliças. 20 ed. Viçosa: UFV, 2000. 402 p.

GALLO, D.; NAKANO, O.; SILVEIRA NETO, S.; CARVALHO, R.P.L.; BATISTA, G.C.; BERT FILHO, E.; PARRA, J.R.P.; ZUCCHI, R.A.; ALVES, S.B.; VENDRAMIM, J.D.; MARCHINI, L.C.; LOPES, J.R.S.; OMOTO, C. Manual de Entomologia Agrícola. Piracicaba: FEALQ. 2002. $920 \mathrm{p}$

GONCCALVES, P.A.S. Flutuação populacional de tripes, Thrips tabaci Lind., em cebola em Ituporanga, Santa Catarina. Anais da Sociedade Entomológica do Brasil. Londrina, v.26, n.2, p.365-369, 1997.

KIMATI, H., AMORIM, L., BERGAMIM FILHO, A ., CAMARGO, L.E.A ., REZENDE, J.A M. Manual de Fitopatologia, Doenças das plantas cultivadas. 3 ed. São Paulo: Ceres, 1997. 774 p.

LALL, B.L.; SINGH, L.M. Biology and control of onion thrips. Journal Economic Entomology, v.61, p.676-679, 1968.

SHELTON, A.M.; NYROP, J.P.; NORTH, R.C.; PETZOLDT, C.; FOSTER, R. Development and use of a dynamic sequential sampling program for onion thrips, Thrips tabaci (Thysanoptera: Thripidae), on onions. Journal Economic Entomology, v.80, p.1051-1056, 1987.

ZAMBOLIM, L., VALE, F.X.R., COSTA, H. Controle de doenças de plantas-hortaliças. Viçosa, 2000, v.1, 444 p. 